\title{
EPOS2012 has better specificity compared to IDSA2012 for diagnosing acute bacterial rhinosinusitis ${ }^{*, \#}$
}

\author{
Kachorn Seresirikachorn ${ }^{1,2}$, Kornkiat Snidvongs ${ }^{1,2}$, Wirach Chitsuthipakorn ${ }^{3}$, \\ Winyu Ruksakul ${ }^{4}$, Supinda Chusakul',2, Jesada Kanjanaumporn 1,2, Songklot \\ Aeumjaturapat ${ }^{1,2}$ \\ ' Department of Otolaryngology, Faculty of Medicine, Chulalongkorn University, Bangkok, Thailand \\ 2 Endoscopic Nasal and Sinus Surgery Excellent Center, King Chulalongkorn Memorial Hospital, Bangkok, Thailand \\ ${ }^{3}$ Department of Otolaryngology, Sawanpracharak Hospital, Nakhonsawan, Thailand \\ ${ }^{4}$ Department of Otolaryngology, Nakhonpathom Hospital, Nakhonpathom, Thailand
}

Rhinology 56: 3, 241-244, 2018

https://doi.org/10.4193/Rhin17.261

*Received for publication:

December 24, 2017

Accepted: January 25, 2018

Background: Acute bacterial rhinosinusitis (ABRS) is a subtype of acute rhinosinusitis (ARS). To prevent excessive antibiotic prescribing, clinical criteria for diagnosing ABRS are presented in two major international guidelines from European Position Paper on Rhinosinusitis and Nasal Polyps (EPOS2012) and the Infectious Diseases Society of America (IDSA2012). This study aims to assess accuracy of these criteria.

Methodology: Patients with ARS were recruited. Clinical features were collected including discolored nasal discharge, facial pain, fever, double sickening, symptoms persisting longer than 10 days, and elevated serum C reactive protein (CRP) and erythrocyte sedimentation rate (ERS). Using middle meatal bacterial culture as a reference, accuracy of EPOS2012 and IDSA2012 criteria were analyzed.

Results: Eighty-eight patients (age $43.2 \pm 14.5$ years, $67 \%$ female) with ARS were recruited. Using the two criteria for diagnosing ABRS, EPOS2012 and IDSA2012 have sensitivity of 50\% (95\%Cl: 38\%-62\%) versus $69 \%$ (95\%Cl: 57\%-79\%), specificity of $63 \%$ (95\%Cl: $43 \%-79 \%$ ) versus $46 \%$ (95\% Cl: $28 \%-65 \%$ ), and accuracy of $53 \%$ versus $63 \%$, respectively.

Conclusion: Both EPOS2012 and IDSA2012 had modest accuracy. EPOS2012 had less sensitivity but a better specificity compared to IDSA2012. This suggests that IDSA2012 diagnostic criteria may contribute to inappropriate use of antibiotics due to poorer specificity.

Key words: sinusitis, bacteria, sensitivity, specificity, accuracy

\section{Introduction}

Acute rhinosinusitis (ARS) is divided into acute bacterial rhinosinusitis (ABRS) and acute viral rhinosinusitis ${ }^{(1)}$. Since it is difficult to differentiate by purely clinical findings between the two subtypes, this contributes to excessive prescription of antibiotics ${ }^{(2)}$. The gold standard in diagnosing ABRS is to cultivate bacteria from sinus cavities. This is not routinely performed because of requirement for special staff, instruments, laboratory access, time loss and significantly greater cost and lack of acceptance by patients. There have been attempts by international groups to establish guidelines for diagnosing ABRS by clinical features alone including the European Position Paper on Rhinosinusitis and Nasal Polyps 2012 (EPOS2012)(1) and Infectious Diseases Society of America (IDSA2012) $)^{(3)}$.

Current definition of ABRS according to EPOS2012 are: rhinosinusitis is less than 12 weeks duration, with increase in symptoms after 5 days or persistent symptoms after 10 days and at least 3 other symptoms and signs: 1 ) discoloured discharge (with unilateral predominance) and purulent secretion in the nasal cavity, 2) severe local pain (with unilateral predominance), 3) fever gre- 
ater than $38^{\circ} \mathrm{C}$, 4) elevated serum $\mathrm{C}$ reactive protein (CRP) and/or erythrocyte sedimentation rate (ESR), and 5 ) double sickening ${ }^{(1)}$. Current definition of ABRS according to IDSA2012 are: rhinosinusitis with one of these three symptoms: 1) onset with persistent symptoms lasting 10 days without any improvement, 2) onset with severe symptoms, characterized by high fever of at least $39^{\circ} \mathrm{C}$ and purulent nasal discharge for at least 3 to 4 consecutive days at the beginning of illness, or 3 ) double-sickening after 5-6 days $^{(3)}$.

Accuracy of the two guidelines in diagnosing ABRS should be different. While EPOS 2012 guidelines will not diagnoses ABRS for patients having symptoms for less than 5 days; IDSA2012 may suggests that some patients have ABRS if their initial symptoms are very severe ${ }^{(3)}$. In addition, laboratory criteria are only used by EPOS2012. EPOS2012 suggests elevation of serum CRP and/or ESR should be a criterium for ABRS(1). Our study aimed to assess sensitivity, specificity and accuracy of EPOS2012 and IDSA2012 for diagnosing ABRS.

\section{Materials and methods}

Patients presented with ARS at the King Chulalongkorn Memorial Hospital, Sawanpracharak Hospital and Nakornpathom Hospital during June 2015 to September 2016 were recruited. Inclusion criteria were: 1) patients with ARS using a diagnostic criteria recommended by the guideline of Acute Rhinosinusitis by The American Academy of Otolaryngology-Head and Neck Surgery ${ }^{(4)}$, and 2) age between 18-65 years. Exclusion criteria were: 1 ) treated with antibiotics, corticosteroids, nonsteroidal anti-inflammatory drugs for any cause within the past 2 weeks, 2) who had received paracetamol within 6 hours, 3) history of ear, neck and nasal cancer, 4) patients who declined participation. They were provided with all advance technical and administration details and with ample time to ask questions which included potential risks and benefits from the study. An informed consent was signed by all volunteers. The study was approved by the Institutional Review Board of the Faculty of Medicine, Chulalongkorn University (number 112/58).

Clinical data collection including discolored discharge, facial pain, fever, double sickening, persistent symptoms for $>10$ days, elevated serum CRP and/or ESR.

Middle meatal bacterial culture were obtained endoscopically and collected with a cotton swab and transported in media to cultivate aerobic bacteria. Positive culture was reported if the quantitative report had $\geq 104$ colony-forming units per milliliter(5) or the semi-quantitative report stated heavy or numerous growth. The sensitivity, specificity, and accuracy of the two criteria were determined. We identified all bacteria cultured but we also focused on patients who had positive cultures of four major common pathogens (i.e. Streptococcus pneumoniae, HaemophiIus influenzae, Moraxella catarrhalis, and Staphylococcus aureus). In addition, sensitivity, specificity, accuracy, and odds ratio of the
Table 1. Number of patients diagnosed as ABRS by using EPOS 2012 and IDSA 2012 and by positivity of any bacteria using middle meatal bacterial culture for diagnosing $A B R S$.

\begin{tabular}{|lcccc|}
\hline & \multicolumn{2}{c}{$\begin{array}{c}\text { Diagnosing ABRS caused by any } \\
\text { Positive } \\
\text { bulture }\end{array}$} & $\begin{array}{c}\text { Tegative } \\
\text { culture }\end{array}$ & \\
\hline EPOS2012 & ABRS & 32 & 9 & 41 \\
\hline Total & no ABRS & 32 & 15 & 47 \\
\hline IDSA2012 & ABRS & 44 & 24 & 88 \\
\hline Total & no ABRS & 20 & 13 & 57 \\
\hline
\end{tabular}

Footnote ABRS: acute bacterial rhinosinusitis.

individual criteria for diagnosing ABRS were assessed.

\section{Statistical analysis}

Statistical analyses were performed using SPSS v 17.0 (Statistical Package for the Social Sciences, Chicago, IL, USA). A difference was considered statistically significant when the two-tailed $P$ value was less than or equal to 0.05 . A four-fold table test was used to calculate for the sensitivity, specificity, and accuracy of EPOS2012 and IDSA2012 in diagnosing ABRS.

\section{Results}

There was a total of 88 patients with ARS participating in the study. There was 59 females (67\%). The average age was $43.2 \pm$ 14.5 years. Duration of ARS was $15.3 \pm 13$ days. The bacteria culture was positive in 64 patients (72.7\%). Of the 64 subjects with positive cultures, 31 (48.4\%) had the common key organisms causing ABRS: Streptococcus pneumoniae, Haemophilus influenzae, Moraxella catarrhalis and Staphylococcus aureus.

Diagnosing ABRS using EPOS2012 and IDSA2012 had overall sensitivities of $50 \%$ (95\%Cl: $38 \%-62 \%$ ) versus $69 \%$ (95\%Cl: $57 \%$ $79 \%)$, specificity of $63 \%$ (95\%Cl: $43 \%-79 \%)$ versus $46 \%(95 \% \mathrm{Cl}$ : $28 \%-65 \%)$, and accuracy of $53 \%$ versus $63 \%$, respectively. Data is displayed in Table 1.

Diagnosing ABRS caused by S. Pneumoniae, H. Influenzae, $M$. Catarrhalis and S. Aureus, had a sensitivity of 42\% (95\%Ci: $26 \%$ $59 \%$ ) versus $61 \%$ (95\%Cl: $44 \%-76 \%)$, specificity of $51 \%(95 \% \mathrm{Cl}$ : $38 \%-63 \%$ ) versus $33 \%$ (95\% Cl: $22 \%-46 \%$ ), and accuracy of $48 \%$ versus 43\%, respectively. Data is displayed in Table 2. When individual criteria used in EPOS2012 and IDSA2012 guidelines were analyzed, discolored discharge was the most sensitive symptom (81\%). Fever was the most specific symptom ( $83 \%$ for fever $>38^{\circ} \mathrm{C}$ and $92 \%$ for fever $>39^{\circ} \mathrm{C}$ ). When odds ratio 
Table 2. Number of patients diagnosed as ABRS by using EPOS 2012 and IDSA 2012 and by positivity of S.pneumoniae, H.influenzae, M.catarrhalis, S.aureus using middle meatal bacterial culture for diagnosing ABRS .

\begin{tabular}{|lcccc|}
\hline & & \multicolumn{2}{c}{$\begin{array}{c}\text { Diagnosing ABRS caused by key } \\
\text { bacteria } \\
\text { Positive } \\
\text { culture }\end{array}$} & $\begin{array}{c}\text { Togative } \\
\text { culture }\end{array}$ \\
\hline EPOS2012 & ABRS & 13 & 28 & 41 \\
\hline Total & no ABRS & 18 & 29 & 47 \\
\hline IDSA2012 & ABRS & 19 & 57 & 88 \\
\hline Total & no ABRS & 12 & 38 & 51 \\
\hline
\end{tabular}

Footnote ABRS: acute bacterial rhinosinusitis.
Table 3. Sensitivity, specificity, accuracy, odd ratio of individual criteria for diagnosing ABRS.

\begin{tabular}{|lcccc|}
\hline & $\begin{array}{c}\text { Sensi- } \\
\text { tivity }\end{array}$ & $\begin{array}{c}\text { Speci- } \\
\text { ficity }\end{array}$ & Accuracy & Odd ratio \\
\hline Discolored discharge & $81 \%$ & $21 \%$ & $65 \%$ & $\begin{array}{c}1.14 \\
(0.36-3.67)\end{array}$ \\
\hline Severe local pain & $59 \%$ & $54 \%$ & $58 \%$ & $\begin{array}{c}1.73 \\
(0.67-4.45)\end{array}$ \\
\hline Fever $>38^{\circ} \mathrm{C}$ & $5 \%$ & $83 \%$ & $26 \%$ & $\begin{array}{c}0.25 \\
(0.05-1.20)\end{array}$ \\
\hline Elevated ESR/CRP & $69 \%$ & $33 \%$ & $59 \%$ & $\begin{array}{c}1.10 \\
(0.40-2.99)\end{array}$ \\
\hline $\begin{array}{l}\text { Double sickening } \\
\text { Persistent symptoms }\end{array}$ & $45 \%$ & $67 \%$ & $51 \%$ & $\begin{array}{c}1.66 \\
(0.62-4.42)\end{array}$ \\
\hline Fever $>399^{\circ} \mathrm{C}$ & $63 \%$ & $38 \%$ & $56 \%$ & \begin{tabular}{c}
1.00 \\
\hline
\end{tabular} \\
\hline
\end{tabular}

Footnote ABRS: acute bacterial rhinosinusitis. was assessed, no significance was found for any criteria. Data is displayed in Table 3.

\section{Discussion}

To the best of our knowledge, this is the first study assessing sensitivity, specificity, and accuracy of the two international guidelines. Our results found that IDSA2012 had better sensitivity than EPOS2012 (69\% versus 50\%). Patients with symptoms of less than 5 days, were diagnosed with ABRS by the IDSA2012 when their symptoms during the first 3-4 days were severe ${ }^{(3)}$. EPOS2012 suggests that ABRS patients should have persistent symptoms for at least 5 days, so that it becomes less sensitive. However, IDSA2012 had less specificity than EPOS2012 (46\% versus 63\%). This may be because IDSA2012 does not use laboratory investigation as criteria. EPOS2012 is more specific because the guideline uses all clinical presentations including presenting symptoms (discoloured discharge, severe local pain), examination (purulent secretion in the nasal cavity, fever), laboratory (CRP, ESR), disease progression (double sickening) and a duration of $>5$ days ${ }^{(1)}$.

Both EPOS2012 and IDSA2012 had modest accuracy. However, in practice, sensitivity and specificity of diagnostic tool are more practical. Suitable criteria for diagnosing ABRS require either high sensitivity or high specificity. The clinical applicability of our findings depends on how physicians aim to use the guidelines. In case physicians would prefer a screening tool for diagnosing ABRS, IDSA2012 may be used. If physicians are looking for criteria to appropriately select cases for prescribing antibiotics, EPOS2012 might be more appropriate. The authors suggest that EPOS2012 should be routinely used in clinical practice due to its better specificity. The use of IDSA2012 in routine practice may contribute to excessive use of antibiotics. Physicians should be aware of prevalence of ARS in the area especially when exposure to predisposing factors of ARS, e.g. smoking is common in the population. Increased prevalence may affect positive predictive value of diagnosing criteria. However, increased prevalence does not affect sensitivity and specificity.

The authors also analyzed the individual criteria for diagnosing ABRS, although many of these criteria had been assessed in previous studies. In contrast to other studies, we found that the odds ratio was not significant for any individual criteria. This contrast may be because references used for diagnosing ABRS are heterogeneous. Gwaltney et al. ${ }^{(6)}$ performed an observational study assessing patients with ARS caused by rhinovirus. Wald et al. ${ }^{(7)}$ performed a descriptive study without data analysis. Berg et al. ${ }^{(8)}$, Hansen et al. ${ }^{(9)}$, Van Buchem et al. ${ }^{(10)}$, Lindbaek et al. ${ }^{(11)}$, Shaikh et al. ${ }^{(12)}$ assessed parameters for diagnosing ARS, other than ABRS. Evan et al. ${ }^{(13)}$ used positive microscopic findings from maxillary tapping as a reference without sending specimen for bacterial culture. Young et al. ${ }^{(14)}$ used positive response to antibiotics as a reference without sending specimen for bacterial culture. Soderstrom et al. ${ }^{(15)}$ and Lacroix et al. ${ }^{(16)}$ used bacterial culture from nasopharynx as a reference. Hansen et al. focused on the maxillary sinus for diagnosing $\mathrm{ABRS}^{(17)}$. Compared to previous studies, we used positive middle meatal bacterial cultures which had to the best of our knowledge never been used. One limitation of this study is that endoscopic middle meatal culture is not considered a gold standard in diagnosing of ABRS. Positive bacterial culture from sinus punctures are generally recommended. However, the authors did not use antral puncture, that requires an invasive procedure, and because the most common source of ABRS has been anterior ethmoid sinuses. 
Negative bacterial culture from specimen collected from maxillary sinus does not diagnose acute bacterial ethmoiditis. In contrast, endoscopic middle meatal samples collected by swab discharge draining from all anterior paranasal sinuses should miss less numbers of patients with ABRS. According to the study by Benninger et al., an endoscopic middle meatal culture could be used to replace puncturing cavities ${ }^{(18)}$. It has a sensitivity of $80.9 \%$, and a specificity of $90.5 \%$. The other limitation is that the authors only performed aerobic cultures and patients with ABRS caused by anaerobic bacteria may have been missed.

\section{Conclusion}

For diagnosing acute bacterial rhinosinusitis, both EPOS2012 and IDSA2012 had modest accuracy. EPOS2012 had less sensitivity but better specificity compared to IDSA2012. In practice, the use of IDSA2012 may contribute to excessive use of antibiotics due to its poor specificity. When the aim is to limit use of antibi- otics, EPOS2012 might be more appropriate.

\section{Acknowledgements}

The authors wish to thank Ratchadapiseksompotch Research Fund for the financial support.

\section{Authorship contribution}

KSE : Study design, data collection, data analysis, manuscript preparation; KSN: Study design, data analysis, manuscript review; WC, WR: Data collection; SC, JK: Manuscript review, SA: Study design, conceptual, manuscript review.

\section{Conflict of interest}

This project Ratchadapiseksompotch Fund, Faculty of Medicine, Chulalongkorn University. Kornkiat Snidvongs has served on speaker's bureau for Merck Sharp Dohme, GlaxoSmithKline and Mylan.

\section{References}

1. Fokkens WJ, Lund VJ, Mullol J, et al. EPOS 2012: European position paper on rhinosinusitis and nasal polyps 2012. A summary for otorhinolaryngologists. Rhinology 2012:50:1-12

2. Anand VK. Epidemiology and economic impact of rhinosinusitis. Ann Otol Rhino Laryngol Suppl 2004;193:3-5.

3. Chow AW, Benninger MS, Brook I, et al. IDSA clinical practice guideline for acute bacterial rhinosinusitis in children and adults. Clin Infect Dis 2012:54:e72-e112.

4. Report of the Rhinosinusitis Task Force Committee Meeting. Alexandria, Virginia August 17, 1996. Otolaryngol Head Neck Surg 1997;117:S1-68.

5. Clinical practice guideline: management of sinusitis. Pediatrics. 2001;108:798-808.

6. Gwaltney JM, Jr., Hendley JO, Simon G, Jordan WS, Jr. Rhinovirus infections in an industrial population. II. Characteristics of illness and antibody response. JAMA 1967;202:494-500.

7. Wald ER, Milmoe GJ, Bowen A, LedesmaMedina J, Salamon N, Bluestone CD. Acute maxillary sinusitis in children. N Engl J Med 1981;304:749-54.

8. Berg O, Carenfelt C. Analysis of symptoms and clinical signs in the maxillary sinus empyema. Acta Otolaryngol 1988;105:3439.

9. Hansen JG, Schmidt H, Rosborg J, Lund E.
Predicting acute maxillary sinusitis in a general practice population. BMJ 1995;311:2336.

10. van Buchem $L$, Peeters $M$, Beaumont J, Knottnerus JA. Acute maxillary sinusitis in general practice: The relation between clinical picture and objective findings. Eur J Gen Pract 1995;1:155-60.

11. Lindbaek M, Hjortdahl P, Johnsen UL. Use of symptoms, signs, and blood tests to diagnose acute sinus infections in primary care: comparison with computed tomography. Fam Med 1996;28:183-8.

12. Shaikh N, Hoberman A, Kearney DH, et al. Signs and symptoms that differentiate acute sinusitis from viral upper respiratory tract infection. Pediatr Infect Dis J 2013;32:1061-5.

13. Evans FO, Jr., Sydnor JB, Moore WE, et al. Sinusitis of the maxillary antrum. N Engl J Med 1975;293:735-9.

14. Young J, Bucher $H$, Tschudi P, Periat $P$, Hugenschmidt C, Welge-Lussen A. The clinical diagnosis of acute bacterial rhinosinusitis in general practice and its therapeutic consequences. J Clin Epidemiol 2003:56:377-84

15. Soderstrom M, Blomberg J, Christensen $P_{\text {, }}$ Hovelius B. Erythromycin and phenoxymethylpenicillin (penicillin $V$ ) in the treatment of respiratory tract infections as related to microbiological findings and serum C-reactive protein. Scand J Infect Dis
1991;23:347-54

16. Lacroix JS, Ricchetti A, Lew D, et al. Symptoms and clinical and radiological signs predicting the presence of pathogenic bacteria in acute rhinosinusitis. Acta Otolaryngol 2002;122:192-6.

17. Hansen JG, Hojbjerg T, Rosborg J. Symptoms and signs in culture-proven acute maxillary sinusitis in a general practice population. APMIS 2009;117:724-9.

18. Benninger MS, Payne SC, Ferguson BJ, Hadley JA, Ahmad N. Endoscopically directed middle meatal cultures versus maxillary sinus taps in acute bacterial maxillary rhinosinusitis: a meta-analysis. Otolaryngol Head Neck Surg 2006;134:3-9.

Kachorn Seresirikachorn

Faculty of Medicine

Chulalongkorn University

Pathumwan

Bangkok10330

Thailand

Tel: +6622564103

Fax: +6622527787

E-mail:kachorns@gmail.com 